\title{
Evaluation of a duodenal digesta sampling system
}

\author{
PH Robinson ${ }^{1}, \mathrm{M}$ Gill 2 \\ ${ }^{1}$ Fredericton Research Centre, PO Box 20280, Fredericton, NB, E3B 4Z7, Canada ; ${ }^{N}$ atural \\ Resources Institute, Chatham Maritime, Chatham, Kent, ME4 4TB, England
}

The standard method for measuring duodenal flow in ruminants with simple gutter-type $T$. piece cannulae, where representative digesta samples cannot be assumed, has been the dual marker system with time sequence sampling (Faichney, 1980, J Agric Sci, 94, 313318). Indigestible markers are chosen for their ability to associate with particulate and liquid phases and the ratio of their concentrations in duodenal digesta, relative to that administered, is used to reconstitute representative digesta samples. While the ability of this procedure to reconstitute digesta samples with the correct liquid to solid ratio has not been questioned, Robinson and Kennelly (1990, J Dairy Sci, 73, 3146-3157) challenged the procedure's ability to reconstitute samples with the correct particle composition. However, an attempt by these researchers to validate the ability of their $T$ piece cannula to collect representative digesta samples was unsuccessful, as digesta flow estimates based upon Co-EDTA and $\mathrm{Cr}$ mordant varied (Table). It was unclear if failure was due to the inability of the cannula to provide representative digesta samples, or the infrequent (every $6 \mathrm{~h}$ ) dosing of indigestible markers which would tend to underestimate the average Co concentration of digesta due to rapid rumen outflow of Co-EDTA and the infrequent (5 samples) digesta sampling schedule.

In the present study, four late lactation dairy cows fitted with these duodenal cannulae, were fed a mixed ration at $08: 00 \mathrm{~h}$ and 18:00 $\mathrm{h}$ and supplemented with a high protein (48\% CP) concentrate, at $15 \%$ of mixed ration DM intake, at either 08:30 h (Day) or 00:30 h (Night). Indigestible markers were either continuously infused (Co-EDTA) or manually dosed to the rumen ( $\mathrm{Cr}$-mordant) at $2 \mathrm{~h}$ intervals. Duodenal digest, 24 samples, were collected at $3 \mathrm{~h}$ intervals, with $23 \mathrm{~h}$ breaks after samples 8 and 16 , in order to provide samples representative of each hour of the day. Individual, as well as bulked samples, were assayed for $\mathrm{Co}$ an $\mathrm{Cr}$ and duodenal DM flow calculated for the day (bulked sample) or for each hour of the day (individual samples).

Results show that daily duodenal DM flow estimates did not differ due to marker for bulked vs hourly samples (Table). This would appear to validate the ability of this cannula/marker system to accurately estimate daily duodenal DM flow. However the trend $(P=0.154)$ to higher duodenal $D M$ flow for hourly estimates, particularly for $\mathrm{CO}$ based estimates, appeared to result from failure of the assumption that marker flow past the duodenum per hour was equal to $1 / 24$ of daily flow. Results of this study show that if representative samples can be obtained daily duodenal DM flow can be estimated using one marker. It is suggested that estimation of hourly flow should be checked by infusion of a marker into the abomasum.

\begin{tabular}{lccc} 
& Previous study ${ }^{1}$ & \multicolumn{2}{c}{ Current study $^{2}$} \\
& & Day & Night \\
$\begin{array}{l}\text { DM intake }(\mathrm{kg} / \mathrm{d}) \\
\text { Duodenal DM flow }(\mathrm{kg} / \mathrm{d})\end{array}$ & 19.11 & 15.94 & 16.92 \\
$\begin{array}{l}\text { Cr-based estimates } \\
\text { Bulked sample }\end{array}$ & & & \\
$\quad$ Hourly samples & 9.72 & 11.01 & 10.51 \\
Co-based estimates & ND & 11.52 & 11.64 \\
$\quad$ Bulked sample & 14.50 & 11.11 & 10.84 \\
Hourly samples & ND & 12.59 & 11.90 \\
\hline
\end{tabular}

1Robinson and Kennelly (1990, J Dairy Sci, 73, 3146-3157)

2Statistical significance : Treatment $P=0.370$, marker (Co vs $C r$ ) $P=0.844$, sample (hourly vs bulked) $P=0.154$, and marker $x$ sample $P=0.550$ 